\title{
Molecular Modeling of 3-(1,3-dioxoisoindolin-2-yl) Benzyl Nitrate and Its Molecular Docking Study with Phosphodiesterase-5 (PDE5)
}

Mehdi Nabati*, Vida Bodaghi-Namileh

Synthesis and Molecular Simulation Laboratory, Chemistry Department, Pars Isotope Company, P.O. Box: 1437663181, Tehran, Iran

\section{ART ICLE INFO}

Received: 10 May 2019

Revised: 14 June 2019

Accepted: 05 July 2019

Available online: 07 July 2019

DOI: $10.33945 /$ SAMI/AJCA.2020.1.7

\section{K E Y W O R D S}

Benzyl nitrate

Molecular docking

Molecular simulation

Phosphodiesterase-5

Sickle cell disease

\section{A B S T R A C T}

In this study, the electronic properties of the novel medicinal compound 3-(1,3-dioxoisoindolin-2-yl) benzyl nitrate as a treatment of sickle cell disease are obtained using density functional theory (DFT) method. In first step, the molecular structure of the title compound is optimized at B3LYP/6-311++G (d,p) level of theory at room temperature. Then, its stability and reactivity properties are calculated by frontier molecular orbitals (FMOs) energies. The global reactivity indices show this medicinal molecule is a more stable compound and the nitrogen atom of the nitrate group has positive charge. So, the nitrate group can quit nitric oxide molecule in binding to Phosphodiesterase-5 (PDE5) enzyme. On the other hand, the docking analysis of the ligand-enzyme complex shows the steric interactions play the main role in this complex formation. Also, the data shows the PDE5 residues containing Phe $[\mathrm{A}]$ 820, Gln [A] 817, Ile [A] 768, Val [A] 782, Gln [A] 775, Phe [A] 786, Ile [A] 778, Leu [A] 765, Met [A] 816, Ala [A] 767 and Tyr [A] 612 play main role in the ligand-enzyme complex formation.

\section{GR A P H I C A L ABS T RAC T}
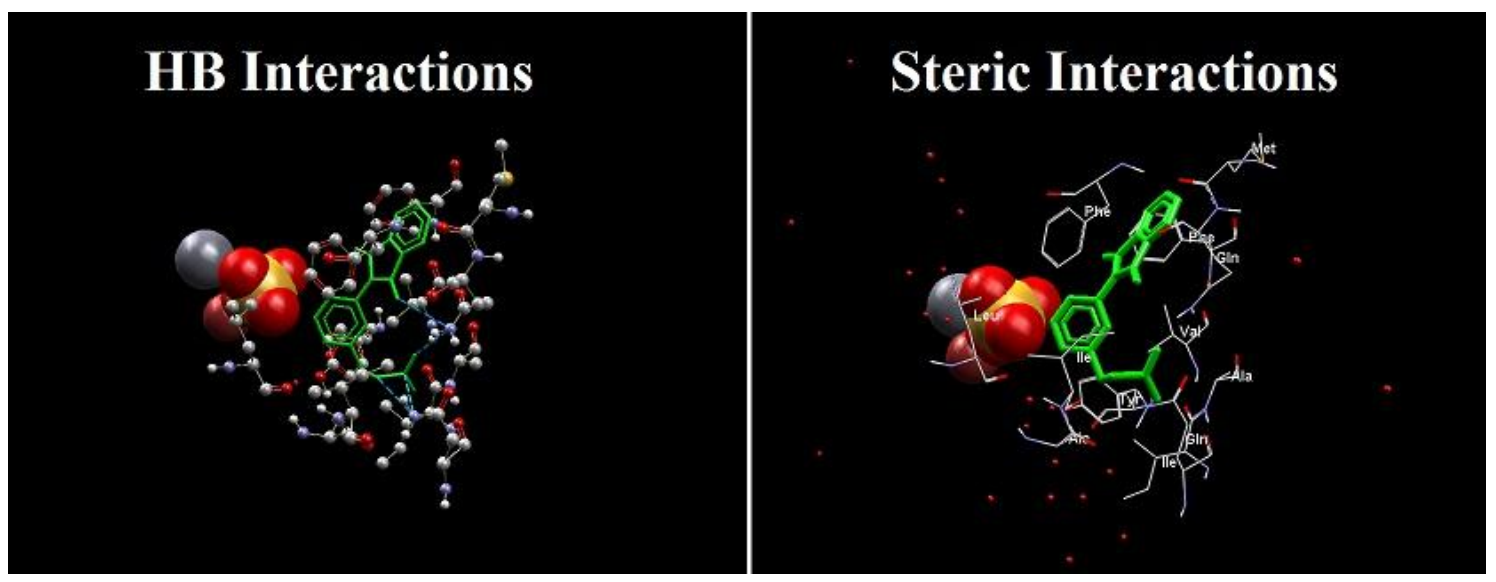

* Corresponding author's E-mail address: mnabati@ymail.com 


\section{Introduction}

Sickle cell disease (SCD) is a genetic disorder in which a mutation of the hemoglobin subunit beta (HBB) results in generation of anomalous hemoglobin in shape of a sickle [1]. Hemoglobin ( $\mathrm{Hb}$ ) is a protein existent in red blood cells and is composed of four subunits responsible for carrying oxygen throughout the body [2]. Each and every major organ in the body is afflicted by SDC and various conditions are associated with this disease. Priapism is one of the conditions occurring in 30 to $45 \%$ of men with SCD due to the dysregulation of genitourinary system [3]. Priapism is a disorder identified by the uncontrollable and persistent penile erection unrelated to sexual stimulation or desire [4]. There are two recognized subtypes of priapism 1) non ischemic or high flow and 2) ischemic or low flow priapism. Patients with SDC experience ischemic priapism and are more likely to undergo more significant complications such as, progressive fibrosis of the penis, necrosis of corpus cavernosum smooth cells and endothelium and subsequently erectile dysfunction (ED) [5]. Generally, there are various mechanisms attributed to the occurrence of priapism including, diminished outflow from penis veins, an increased level of adenosine, the formation of erythrocytes sludge in vasculature of the penis and reduction of phosphodiesterase- 5 (PDE5) due to depleted nitric oxide (NO) levels. SCD-induced priapism comes about as a result of NO deficiency [6]. The major molecular pathway involved in the complex process of normal penile erection is NO/cGMP/PDE5 signaling pathway [7]. Physiologically, NO synthesis in the body is regulated by NO synthase (NOS) specifically, its neuronal (nNOS) and endothelial (eNOS) isoforms. The phosphorylation and consequent activation of these NOS isoforms promote the generation of NO from $L^{-}$ arginine [8]. NO binds to and activates guanylate cyclase (GC) following its absorption by smooth muscle cells. GC then catalyzes the reaction of converting guanosine-5' - triphosphate to cGMP and thus initiates the process of cavernosal smooth muscle relaxation and penile erection. The cessation of penile erection is a result of cGMP inactivation and conversion to $5^{\prime}$-GMP by PDE5 enzyme. PDE5 is an enzyme belonging to PDE superfamily. The mentioned enzymes are in charge of catalyzing the inactivation reaction of cGMP and cAMP. PDE5 mostly breaks down cGMP and is distinctly distributed in smooth muscles of renal tubules [9]. The reduced levels of NO in SCD are recognized as the principal mechanism contributing to the disturbance of this physiological balance and consequent occurrence of priapism. The subsequent decrease in PDE5 expression, results in an uncontrolled activation and accumulation of cGMP and a persistent erection [10]. Considering the key role NO pathway plays in SDC-induced priapism, investigating agents with NO donor properties seems logical. In a study by Silva et al., the NO donor properties of the compound 3-(1,3-dioxoisoindolin-2-yl) benzyl nitrate, synthesized by hydroxyurea and thalidomide molecular hybridization, was evaluated [11].

While this study extensively discusses NO/cGMP/PDE5 pathway in regulating penile erection and SDC-induced priapism and investigates the beneficial effects of 3(1,3-dioxoisoindolin-2-yl) benzyl nitrate, in ameliorating decreased NO bioavailability, the exact molecular mechanism of this compound remains unclear. The present study was undertaken in order to shed some light on NO donor capacity of the titled compound and to achieve a more comprehensive interpretation of the exact underlying mechanism contributing to this capability, using molecular docking 
methods and computational chemistry. Furthermore, the pharmacokinetics and biological attributes of this compound was investigated using Swiss ADME web tool.

\section{Experimental}

\section{Computational methods}

In medicinal chemistry, one of the main parameters is identifying the exact geometrical structure of the active substances [12]. Quantum mechanics (QM) and molecular dynamics (MD) are two important fields of the theoretical chemistry that help us to access the molecular structure of the medicinal compounds [1214]. The quantum mechanical studies give us good responses in calculation of the small molecules [15]. Here, the present research study is related to investigation of the structural and electronic properties of 3-(1, 3-dioxoisoindolin-2-yl) benzyl nitrate (the novel small molecule for priapism treatment) using quantum mechanics field of theoretical chemistry. So, the density functional theory (DFT) method will be used to compute the electronic properties of the said active substance. The molecular structure of the title compound optimizes using B3LYP/6-311++G (d,p) basis set of theory at room temperature in isolated form. To study the reactivity and stability of this compound, the global reactivity indices will be calculated. We use the frontier molecular orbitals (FMOs) theory to access the global reactivity indices. On the other hand, one important field in the simulation of biomolecules is docking of the molecules into the active sites of the enzymes, proteins or receptors. In fact, this method is used to prediction of the preferred orientation of one molecule to an enzyme when bound to each other for a stable complex formation. In this study, we use Molegro Virtual Docker (MVD) software to predict the preferred orientations of 3-(1, 3-dioxoisoindolin-2-yl) benzyl nitrate in the drug-enzyme complex.

\section{Results and discussion}

\section{3-(1,3-dioxoisoindolin-2-yl) benzyl nitrate structural properties study}

The molecular structure of the novel compound 3-(1,3-dioxoisoindolin-2-yl) benzyl nitrate is shown in Scheme 1. This active substance has been made by isoindoline and benzene rings. A nitrate group has been attached to the benzene ring. So, this compound can be a donor group in the bio reactions. As can be said in the computational method section, the title molecular structure was optimized using B3LYP functional and 6-311++G (d,p) level of theory at room temperature in isolated form. Figure 1 indicates the theoretical geometric structure of the said compound. This graph shows the benzene ring and isoindoline system are twisted to each other. Figure 2 indicates the dependence between the theoretical and experimental bond lengths of the compound 3-(1,3dioxoisoindolin-2-yl) benzyl nitrate. This dependence is shown by the equation $y=0.8947 x+0.1164$. The higher correlation coefficient $\left(R^{2}=0.9431\right)$ for this equation shows a great convergence. So, the B3LYP/6-311++G $(d, p)$ basis set of theory is a good method to compute the electronic properties of the title compound.

Stability and reactivity study of the compound 3-(1,3-dioxoisoindolin-2-yl) benzyl nitrate

Frontier molecular orbitals (FMOs) are the orbitals most likely to be involved in reactivity and stability of chemical compounds. The FMOs are divided to two types of orbitals: the highest occupied molecular orbital (HOMO) and the lowest unoccupied molecular orbital (LUMO). 
Scheme 1. 3-(1,3-dioxoisoindolin2-yl) benzyl nitrate molecular structure

Figure 1. The theoretical geometric structure of 3-(1,3dioxoisoindolin-2-yl) benzyl nitrate

Figure 2. The experimental and theoretical bond lengths relationship of 3-(1, 3dioxoisoindolin-2-yl) benzyl nitrate
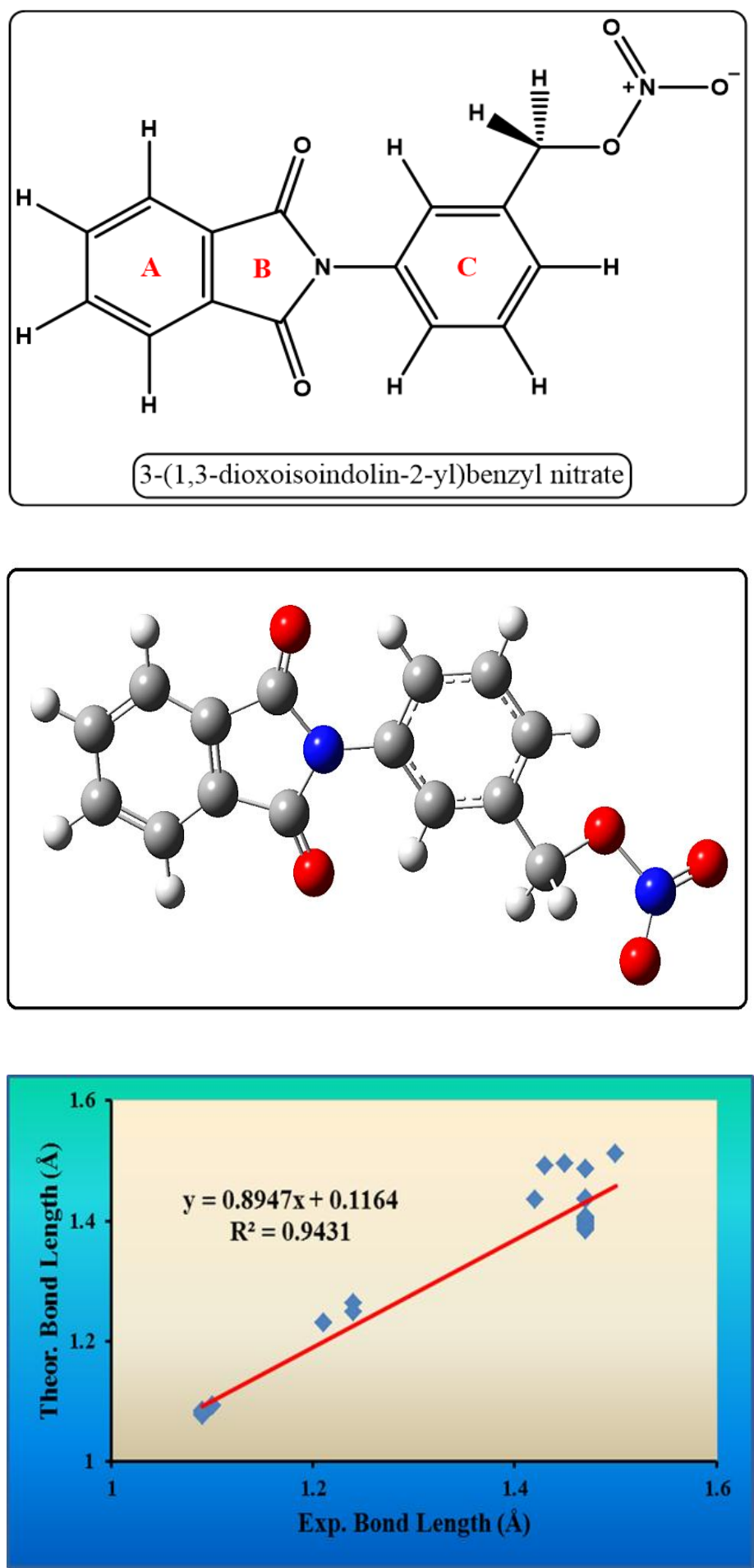

The electrons of HOMO are easily removed in terms of energy. So, these electrons can be simply donated to form a bond. On the other hand, the electrons are easily added to the LUMO. So, this orbital can be acted as a Lewis acid. To happening reaction between two compounds, the HOMO of one compound must overlap with the LUMO of another molecule [16-19]. Figure 3 shows the frontier molecular orbitals (the filled HOMO and the empty LUMO) of the active compound 3-(1,3dioxoisoindolin-2-yl) benzyl nitrate. We can see the highest energy occupied molecular orbital (filled HOMO) is mainly constructed by the elements of the benzene ring, while the lowest energy unoccupied molecular orbital 
(the empty LUMO) has been made by the atoms of the isoindoline system (rings $A$ and $B$ ). It is deduced that the benzene ring will be participated in nucleophilic reactions as an electron donating ring in interaction with the residues of an enzyme. In contrast, the isoindoline system will indicate the electrophilic or electron accepting property in interacton with PDE5 enzyme. Two important physical parameters of one chemical compound are reactivity and stability. The stability and global reactivity indices are used to understand these parameters. The energies of the frontier molecular orbitals (HOMO and LUMO) are used to calculate the global reactivity indices [20]. The global reactivity descriptors like energy gap (Eg), ionization potential (IP), electron affinity (EA), chemical hardness $(\eta)$, chemical softness (S), electronegativity $(\chi)$, electronic chemical potential $(\mu)$ and electrophilicity index $(\omega)$ can be obtained from the energies of the frontier orbitals. These reactivity indices are achieved by following formulas [21]:

$E_{g}=E_{\text {LUМо }}-E_{\text {Номо }}$

$I P=-E_{\text {Номо }}$

$E A=-E_{L U M O}$

$\chi=\frac{-\left(\varepsilon_{L U M O}+\varepsilon_{H O M O}\right)}{2}$

$\mu=\frac{\left(\varepsilon_{L U M O}+\varepsilon_{H O M O}\right)}{2}$

$\omega=\frac{\mu^{2}}{2 \eta}$

$S=\frac{1}{\eta}$

The frontier molecular orbitals (filled HOMO and empty LUMO) energies and global reactivity indices of 3-(1,3-dioxoisoindolin-2yl) benzyl nitrate have been listed in Table 1.
As can be seen from this table, the energies of HOMO and LUMO are $-6.48 \mathrm{eV}$ and $-2.63 \mathrm{eV}$, respectively. The low frontier molecular orbitals energies indicate high molecular structure stability. The HOMO/LUMO energies gap (Eg) is $3.85 \mathrm{eV}$. This HOMO/LUMO energies gap shows the susceptibility of the said compound to react with other reagents. A density of states (DOS) graph shows the density of the frontier molecular orbitals with their energies. In fact, this graph indicates the tendency of the molecular structure to perform nucleophilic and electrophilic reactions [22]. Figure 4 indicates the density of states (DOS) graph of the title compound. It can be seen from this graph that both occupied and unoccupied molecular orbitals have low density. So, it can be deduced that the title active compound has a stable molecular structure and it probably shows high resistance to oxidants and reductants. The low energy of the electron affinity (EA) and high energy of the ionization potential (IP) show the high nucleophilic property of the molecule, too. The electrophilicity of this compound is $5.39 \mathrm{eV}$. The high amount of the electrophilicity index proves the high tendency of the said compound to react with electrophiles. On the other hand, the low chemical hardness and high amount of the chemical softness indicates this electron accepting property of the compound. Another important physical parameter to determining the reactivity of an organic compound is molecular electrostatic potential (MEP) [23]. The electrostatic potentials negative, zero and positive have been shown by red, green and blue colors in molecular electrostatic potential (MEP) graph (Figure 5). It can be seen that all segments of the molecule except the oxygen atoms have electrostatic potential zero or positive. So, the nitrate group of this molecular structure can act as an $\mathrm{N}=\mathrm{O}$ donor. 
Figure 3. The frontier molecular orbitals of 3-(1,3-

dioxoisoindolin-2-yl) benzyl nitrate

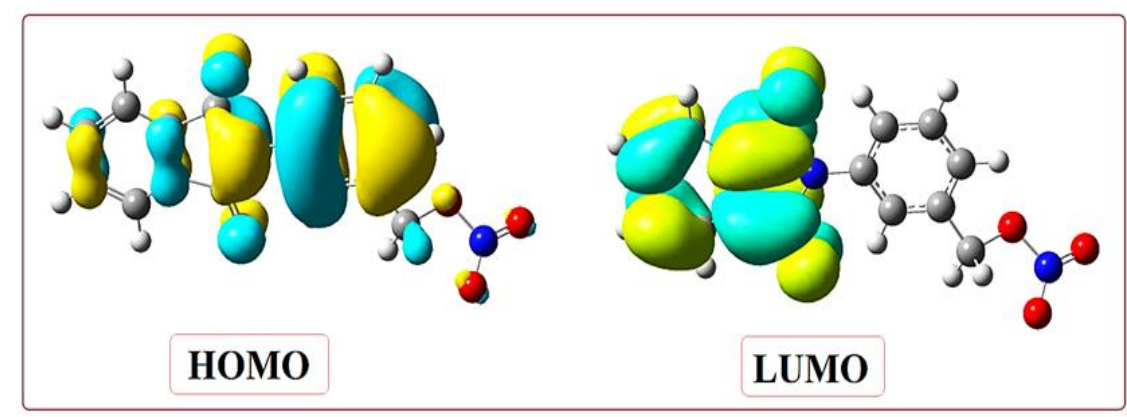

Figure 4. The density of states (DOS) graph of 3-(1,3dioxoisoindolin-2-yl) benzyl nitrate

Figure 5. The molecular electrostatic potential (MEP) graph of 3-(1,3-dioxoisoindolin2-yl) benzyl nitrate
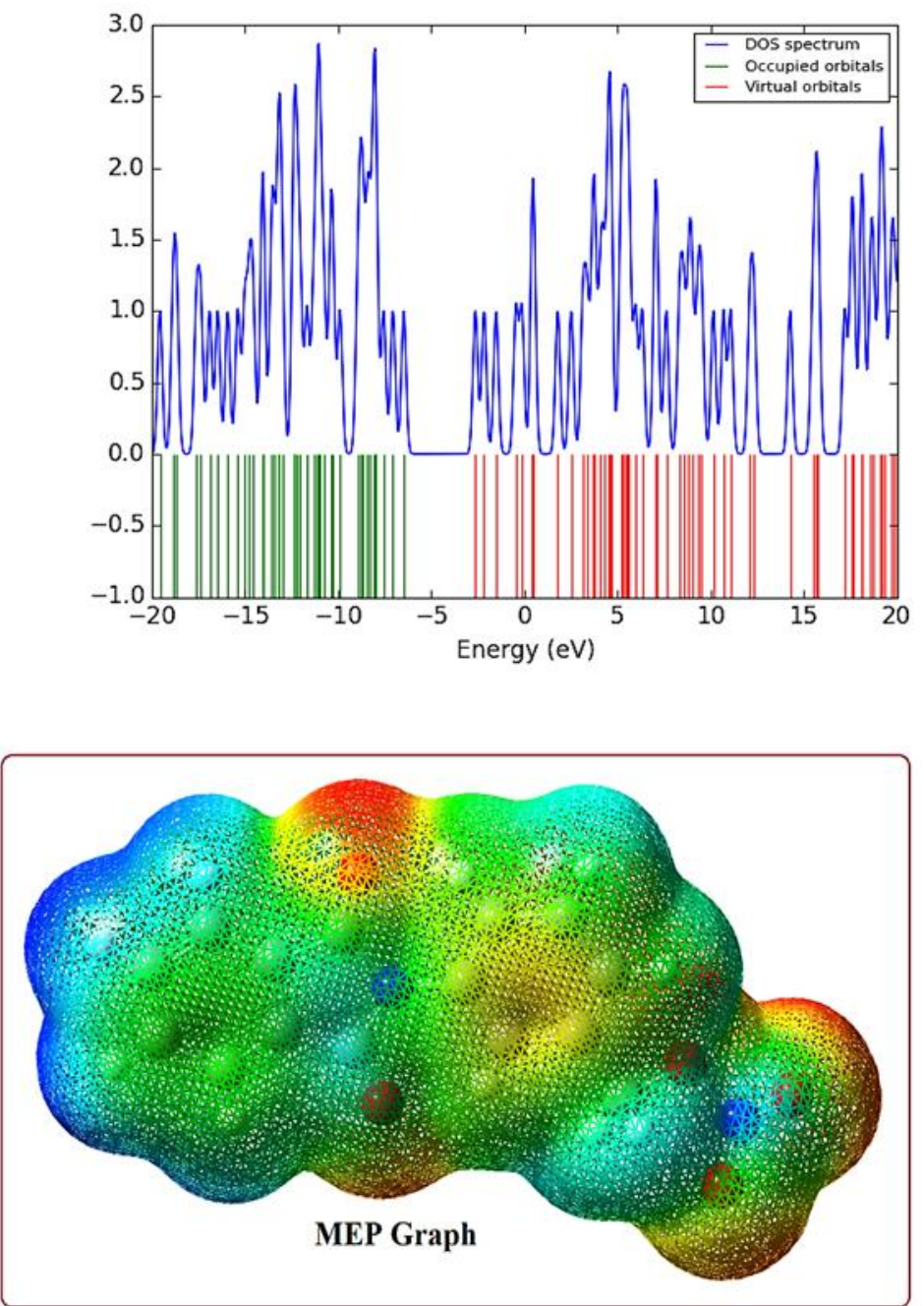

Physicochemical descriptors and ADME parameters of the compound 3-(1,3dioxoisoindolin-2-yl) benzyl nitrate

Ever since the late 1990s, drug discovery and development have relied on analyzing absorption, distribution, metabolism and excretion (ADME) and ADME-related physicochemical parameters to predict the oral bioavailability and drug likeness of molecular compounds [24]. In this study, Swiss ADME web tool was employed for computational analysis of physicochemical descriptors as well as predicting ADME parameters and pharmacokinetic properties of the investigated molecular structure. 
Table 1. Global reactivity indices of 3-(1,3-dioxoisoindolin-2-yl) benzyl nitrate

\begin{tabular}{cc} 
Parameter & Energy value $(\mathrm{eV})$ \\
HOMO & -6.48 \\
LUMO & -2.63 \\
Ionization Potential (IP) & 6.48 \\
Electron Affinity (EA) & 2.63 \\
Energy Gap (Eg) & 3.85 \\
Electronegativity $(\chi)$ & 4.56 \\
Chemical Potential $(\mu)$ & -4.56 \\
Chemical Hardness $(\eta)$ & 1.93 \\
Chemical Softness $(S)$ & 0.52 \\
Electrophilicity index $(\omega)$ & 5.39 \\
\hline
\end{tabular}

Figure 6 indicates the predicted physicochemical graph of the title molecule. The colored zone shows the suitable physicochemical space for oral bioavailability. Physiological properties of this compound include a molecular weight of $298.25 \mathrm{~g} / \mathrm{mol}, 4$ rotatable bonds and 5 hydrogen bond acceptors. The fraction Csp3 of this compound is 0.77 , topological polar surface area (TPSA) is $92.43 \AA^{2}$ and the molar refractivity is 81.12. To predict compound's capacity to permeate through biological membranes, lipophilicity was determined by measuring the partition coefficient between n-octanol and water (log $\mathrm{P}_{0 / \mathrm{w}}$ ). Based on five predictive models, iLog $\mathrm{P}$ of this compound is $1.87, \mathrm{MLog} \mathrm{P}$ is $1.99, \mathrm{XLog}$ $\mathrm{P}$ is $2.47, \mathrm{WLog} \mathrm{P}$ is 1.66 and SILICOS-IT is 0.20 . Predicting water solubility is of great importance in both oral and parenteral pharmaceutical forms as it affects absorption in the former and delivery of active ingredient in the latter. Water solubility of the compound was determined using ESOL model, a topical method to evaluate $\log \mathrm{S}$. The Log S scale is used to predict water solubility. In this regard, a compound could be: insoluble ( $\log S<-10$ ), poorly soluble $(-10<\log S<-6)$, moderately soluble $(-6<\log S<-4)$, soluble $(-4<\log S<-$ $2)$, very soluble $(-4<\log S<-2)$ and highly soluble ( $\log S>0$ ). $\log S$ for the title compound is -3.38 and is thus considered soluble in water. The pharmacokinetic parameters predict individual ADME attributes of the compound under investigation. This molecule possesses a high gastrointestinal (GI) absorption but does not permeate BBB. Furthermore, since metabolic biotransformation of the drug is mediated through CYP 450 isoenzymes, recognizing inhibitory properties of molecular compounds helps predicting the potential drug-drug interactions and adverse effects. The title compound inhibits CYP1A2, CYP2C19 isoforms. The skin permeation index ( $\log \mathrm{Kp}$ ) is related to lipophilicity and size of the molecule and the more negative values are indicative of less skin permeability. This molecule has a Log Kp of $-6.37 \mathrm{~cm} / \mathrm{s}$. Drug likeness is assessed based on bioavailability score and whether the compound obeys Lipinski's rule of five which includes 1) A molecular weight less than 500 Daltons, 2) No more than 5 hydrogen bond donors ( $\mathrm{NH}$ or $\mathrm{OH}), 3$ ) No more than 10 hydrogen bond acceptors ( $\mathrm{N}$ or $\mathrm{O}$ ) and 5) MLog P less than 4.15. The bioavailability score of the compound is 0.55 and it complies with Lipinski's rule. 
Figure 6. The physicochemical properties graph of 3-(1,3-dioxoisoindolin-2-yl) benzyl nitrate

Figure 7. The charge distribution of 3-(1,3dioxoisoindolin-2-yl) benzyl nitrate

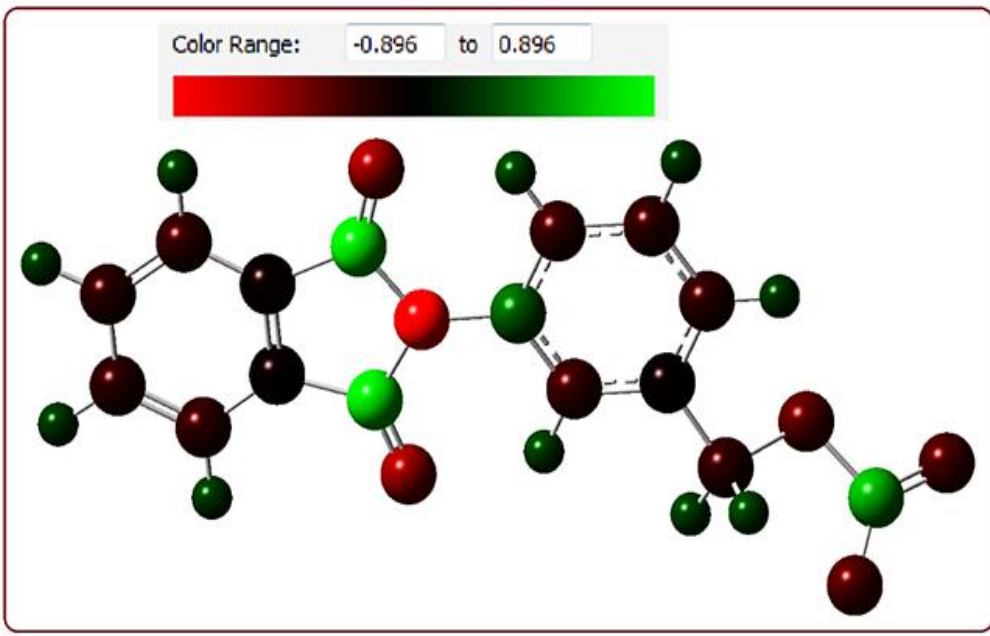

Figure 8. The twodimensional electron localization graph of 3-(1,3dioxoisoindolin-2-yl) benzyl nitrate

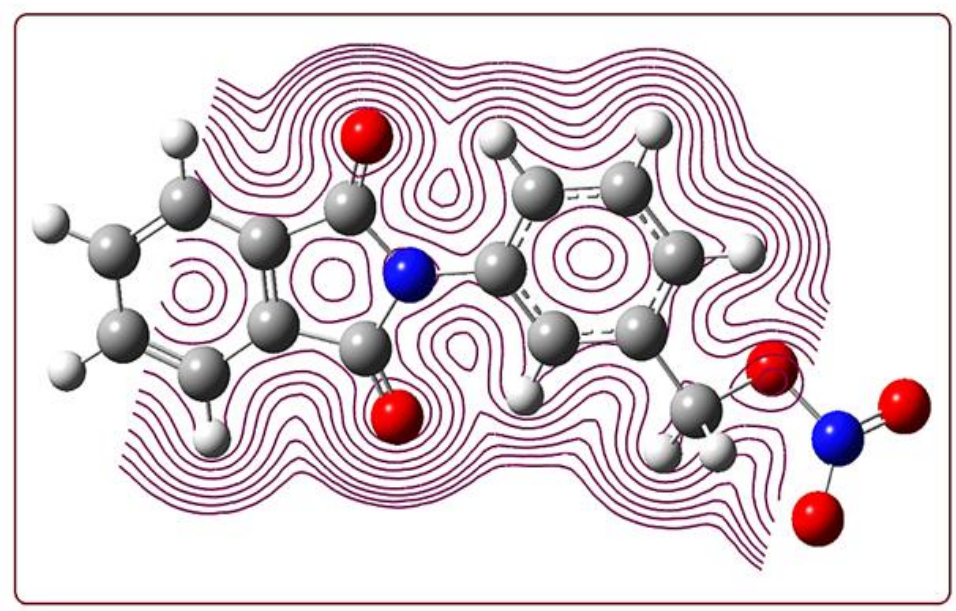

Charge distribution and molecular docking

By specifying the charge density of each of the atoms, we can determine which central one will react to which of the nucleophilic or electrophilic residues of the enzyme [25]. The
Mulliken charge distribution on atoms of the said compound is shown in Figure 7. In this graph, the red, black and green colors are related to the negative, zero and positive charges, respectively. The charge distribution amount on the atoms of the title compound has 
been shown in Figure 8. We can see the carbon atoms of the carbonyl groups and the nitrogen atom of the nitrate group have positive charge. So, these atoms can interact with the atoms containing negative charge. It is concluded that the nitrate group can be interacted with residues of the enzyme and produce an $\mathrm{N}=\mathrm{O}$ molecule. On the other hand, Figure 8 indicates the two-dimensional electron localization graph of 3-(1,3-dioxoisoindolin-2-yl) benzyl nitrate. This graph shows that the main charge localization is around the molecular structure of the title compound. So, this molecule has high tendency to interact with enzyme residues by steric interactions.

Literature review clearly shows that the novel medicinal compound 3- $(1,3-$ dioxoisoindolin-2-yl) benzyl nitrate has high affinity to interact with phosphodiesterase-5 (PDE5) and can quit an $\mathrm{N}=\mathrm{O}$ molecule [11]. So, the binding of 3-(1, 3-dioxoisoindolin-2-yl) benzyl nitrate to phosphodiesterase-5 (PDE5) enzyme and their intearctions analysis are our main purpose in this research article. The three dimensional crystal structure of the title enzyme was obtained from protein data bank (PDB) and the docking analysis was performed using Molegro Virtual Docker (MVD) program. Figure 9 indicates 3-(1,3-dioxoisoindolin-2-yl) benzyl nitrate embedded in the active site of the PDE5 enzyme. From the data of the Table 2, the formation of the molecule-PDE5 complex is mainly done by steric interactions with moldock score -115.475. The hydrogen bond interactions score is -9.549. So, the hydrogen bond interactions have not important role in complex formation of the said active substance with PDE5. On the other hand, the scores of internal ligand interactions (torsional strain and steric interaction) are 6.230 and 9.268, respectively. By considering both internal and external interctions of the compound-PDE5 complex, the total energy score of the system is -112.388 . Our docking analysis shows the steric interactions are the main role in compound-PDE5 complex formation. Figure 10 shows the H-bond and steric interactions of the molecule under study embedded in the active site of the PDE5 enzyme. The residues Gln 775 and Gln 817 of phosphodiesterase-5 interact with the title compound by hydrogen bonds. In contrast, the residues Met 816, Phe 786, Gln 775, Ala 779, Val 782, Ala 767, Ile 778, Ile 768, Gln 817 , Phe 820 , Tyr 612, Leu 765, and Asp 764 are participated in steric interactions with the molecule under study. It can be seen from the data of the Table 3 that the PDE5 residues containing Phe $[A]$ 820, Gln [A] 817, Ile [A] 768, Val [A] 782, Gln [A] 775, Phe $[A]$ 786, Ile $[A]$ 778, Leu [A] 765, Met [A] 816, Ala [A] 767 and Tyr [A] 612 play main role in the ligand-enzyme complex formation. On the other hand, the cofactor $\mathrm{SO}_{4} 2$ of the PDE5 enzyme interacts with the molecule with the energy score -3.222 .
Figure 9. Ligand 3-(1,3dioxoisoindolin-2-yl) benzyl nitrate embedded in the active site of the PDE5 enzyme

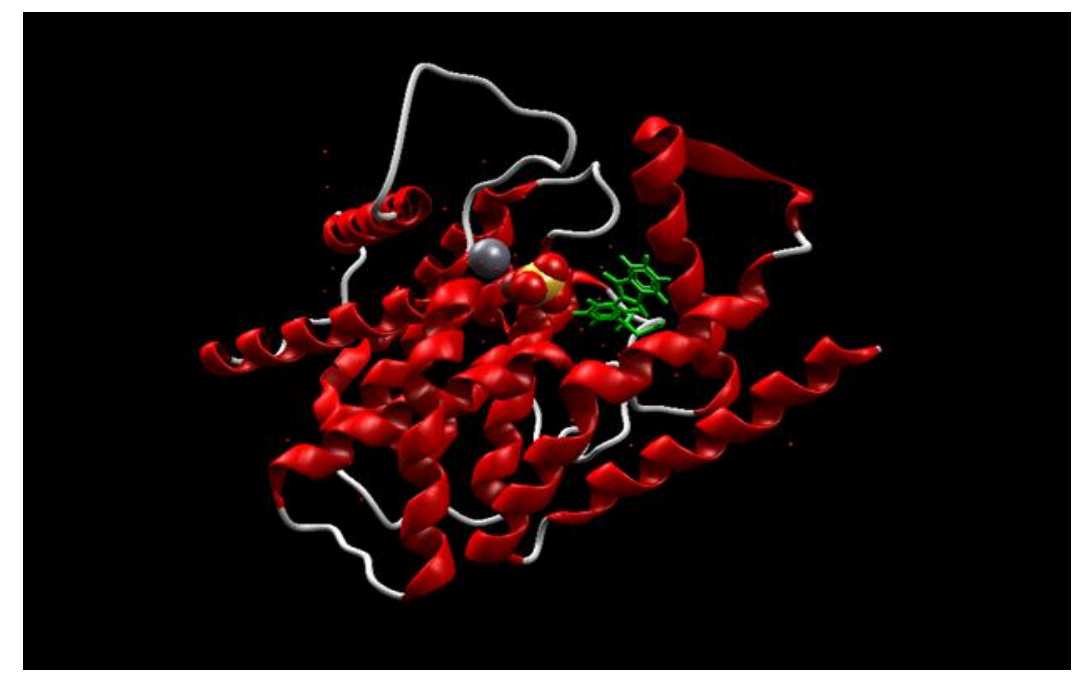



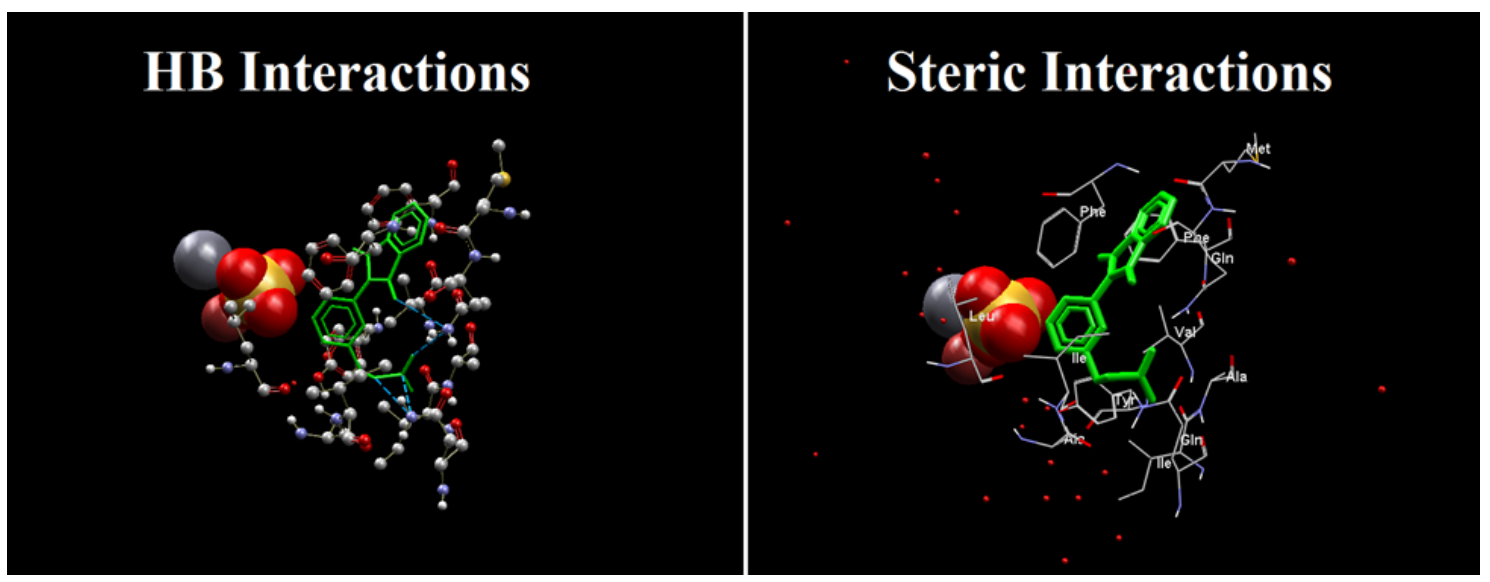

Figure 10. H-bond and steric interactions of ligand 3-(1,3-dioxoisoindolin-2-yl) benzyl nitrate embedded in the active site of the PDE5 enzyme

Table 2. The ligand-PDE5 interactions

\begin{tabular}{|c|c|c|}
\hline \multicolumn{2}{|c|}{ Interactions } & Moldock Score \\
\hline \multirow{4}{*}{ Protein-Ligand Interactions } & Steric (by PLP) & -115.475 \\
\hline & Steric (by LJ12-6) & -38.877 \\
\hline & Hydrogen bonds & -9.549 \\
\hline & Hydrogen bonds (no directionality) & -18.043 \\
\hline \multirow[t]{2}{*}{ Water-Ligand Interactions } & & 0.406 \\
\hline & Torsional strain & 6.230 \\
\hline \multirow[t]{2}{*}{ Internal Ligand Interactions } & Steric (by PLP) & 9.268 \\
\hline & Steric (by LJ12-6) & 54.470 \\
\hline $\begin{array}{l}\text { External and Internal Ligand } \\
\text { Interactions }\end{array}$ & Total Energy & -112.388 \\
\hline
\end{tabular}

Table 3. The participated PDE5 residues in ligand-receptor interactions

\begin{tabular}{cc} 
Residue/HOH & Total energy score \\
Phe [A] 820 & -28.1884 \\
Gln [A] 817 & -17.1132 \\
Ile [A] 768 & -11.1173 \\
Val [A] 782 & -10.3383 \\
Gln [A] 775 & -8.31375 \\
Phe [A] 786 & -7.83284 \\
Ile [A] 778 & -7.77324 \\
Leu [A] 765 & -7.50322 \\
Met [A] 816 & -7.38135 \\
Ala [A] 767 & -6.99233 \\
Tyr [A] 612 & -5.38951 \\
Ala [A] 779 & -3.69772 \\
Cofactor SO4 [A] 2 & -3.22227 \\
Asp [A] 764 & -1.09856 \\
Water HOH [A] 1 & 0.482588 \\
\hline
\end{tabular}




\section{Conclusions}

Reactivity and stability properties study of the novel medicinal compound 3-(1,3dioxoisoindolin-2-yl) benzyl nitrate and its docking with PDE5 enzyme are the main purposes of the present investigation. To attain these chemical and biochemical properties, the molecular structure of the said compound was optimized at B3LYP/6$311++G(d, p)$ basis set of density functional theory and then its binding with PDE5 enzyme was analyzed using MVD software. The ligand-enzyme complex docking analysis shows the steric interactions play the main role in this complex formation. Also, the data shows the PDE5 residues containing Phe [A] 820, Gln [A] 817, Ile [A] 768, Val [A] 782, Gln [A] 775, Phe $[A]$ 786, Ile [A] 778, Leu [A] 765, Met [A] 816, Ala [A] 767 and Tyr [A] 612 play main role in the ligand-enzyme complex formation.

\section{Conflict of interests}

The authors declare that there is no conflict of interests regarding the publication of this paper.

\section{Acknowledgments}

The corresponding author is grateful to Dr. Hamideh Sabahnoo for providing valuable suggestions.

\section{References}

[1]. I. Benenson, S. Porter, Orthop. Nurs., 2018, 37, 221-227.

[2]. G.J. Kato, F.B. Piel, C.D. Reid, M.H. Gaston, K. Ohene-Frempong, L. Krishnamurti, W.R. Smith, J.A. Panepinto, D.J. Weatherall, F.F. Costa, E.P. Vichinsky, Nat. Rev. Dis. Primers., 2018, 4, 18010.

[3]. B. Musicki, S. Karakus, U. Anele, F. Silva, A. Burnett, J. Sex. Med., 2018, 15, S47.
[4]. J.D. La Favor, Z. Fu, V. Venkatraman, T.J. Bivalacqua, J.E. Van Eyk, A.L. Burnett, J. Proteome. Res., 2018, 17, 1031-1040.

[5]. S. AlDallal, N. AlDallal, A. Alam, Cogent Medicine, 2016, 3, 1268357.

[6]. P. Sundd, M.T. Gladwin, E.M. Novelli, Annu. Rev. Pathol-Mech., 2019, 24, 263-292.

[7]. A.J. Nardozza, M.R. Cabrini, Rev. Assoc. Med Bras., 2017, 63, 689-692.

[8]. S. Korkmaz-Icöz, T. Radovits, G. Szabó, Br. J. Pharmacol., 2018, 175, 223-231.

[9]. U.A. Anele, A.L. Burnett, Sexual. Med. Rev., 2015, 3, 160-168.

[10]. B. Musicki, S. Karakus, W. Akakpo, F.H. Silva, J. Liu, H. Chen, B.R. Zirkin, A.L. Burnett, Andrology., 2018, 6, 184-191.

[11]. F.H. Silva, S. Karakus, B. Musicki, H. Matsui, T.J. Bivalacqua, J.L. Dos Santos, F.F. Costa, A.L. Burnett, J. Pharm. Exp. Ther., 2016, 359, 230-237.

[12]. M. Nabati, M. Kermanian, H. Mohammadnejad-Mehrabani, H.R.

Kafshboran, M. Mehmannavaz, S. Sarshar, Chem. Method., 2018, 2, 128-140.

[13]. M. Nabati, M. Mahkam, Org. Chem. Res., 2016, 2, 70-80.

[14]. M. Nabati, J. Phys. Theor. Chem. IAU Iran, 2017, 14, 283-293.

[15]. M. Nabati, Chem. Method., 2017, 1, 121135.

[16]. M. Nabati, J. Phys. Theor. Chem. IAU Iran, 2017, 14, 49-61.

[17]. M. Nabati, H. Sabahnoo, J. Med. Chem. Sci., 2019, 2, 118-125.

[18]. F. Yang, C. Wu, Z. Li, G. Tian, J. Wu, F. Zhu, J. Zhang, Y. He, J. Shen, Org. Process Res. Dev., 2016, 20, 1576-1580.

[19]. M. Nabati, Iran. Chem. Commun., 2019, 7, 324-334.

[20]. M. Nabati, H. Sabahnoo, E. Lohrasbi, M. Mazidi, Chem. Method., 2019, 3, 383-397.

[21]. M. Nabati, Chem. Method., 2018, 2, 223238.

[22]. M. Nabati, Iran. J. Org. Chem., 2018, 10, 2457-2465. 
[23]. M. Nabati, Asian J. Green Chem., 2019, 3, 258-270.

[24]. A. Kumer, N. Sarkar, S. Paul, A. Zannat, Adv. J. Cem. A, 2019, 2, 190-202.

[25]. M. Rahimi, S. Jamehbozorgi, H. Chermette, R. Ghiasi, M. Poor Kalhor, Iran. Chem. Commun., 2019, 7, 472-479.

How to cite this manuscript: Mehdi Nabati*, Vida Bodaghi-Namileh, Molecular Modeling of 3-(1,3dioxoisoindolin-2-Yl) Benzyl Nitrate and Its Molecular Docking Study with Phosphodiesterase-5 (PDE5), Adv. J. Chem. A, 2020, 3(1), 58-69. 